\title{
Self-Adaptive Dynamic Ranging Model-Based Real-Time Hybrid Algorithm for Accurate Indoor Localization
}

\author{
Zhonghui Jiang ${ }^{1}$, Wu Huang ${ }^{2 *}$, Xiao Wei ${ }^{1}$, Defu Cheng ${ }^{1}$, Dan $\mathrm{Li}^{1}$ \\ ${ }^{1}$ Chengdu Techman Software Co., Ltd, Chengdu, China. \\ ${ }^{2}$ Sichuan University, Chengdu, China. \\ * Corresponding author. Tel.:13088058715; email: huangwu@scu.edu.cn \\ Manuscript submitted March 10, 2020; accepted June 1, 2020. \\ doi: 10.17706/ijcce.2020.9.4.167-184
}

\begin{abstract}
The reliability of location information still maintains a crucial impact on restricting the development of location based services in indoor environment. However, in wireless local area network, received signal strength indicator (RSSI) is prone to be interfered by indoor complex environment, resulting in low accuracy and instability of real-time positioning. Here, the new self-adaptive dynamic ranging model-based real-time hybrid algorithm was proposed to realize accurate and undisturbed localization in indoor scenes. A self-adaptive dynamic ranging model was initially constructed to update the environmental parameters and correct the ranging values of mobile terminals in real time. Based on this model, a hybrid KNN algorithm and a hybrid Bayesian algorithm were severally presented. Location fingerprint database and real-time RSSI data of test points were then obtained through data acquisition. Finally, the acquired data was further used to verify the two hybrid algorithms proposed, and compared with the results of several conventional algorithms. As a result, the stability and accuracy of dual hybrid algorithms were better than those of the traditional ones. The range of average location error of both hybrid algorithms maintained 1.26-1.38 m, which was significantly lower than the error level of 2-5 m under the current WLAN environment. This newly proposed hybrid algorithm could effectively improve the stability and accuracy of indoor localization with real-time positioning algorithm, providing a promising solution for RSSI-based indoor positioning system.
\end{abstract}

Key words: Hybrid intelligent systems, indoor environments, received signal strength indicator, simultaneous localization and mapping, wireless LAN.

\section{Introduction}

With the rapid development of the information age and the internet of things, location-based services related to wireless localization technologies have acquired a broad spectrum of applications in various fields [1]-[3]. A global positioning system (GPS) can provide high-precision position information for mobile terminals, especially in outdoor positioning [4]-[6]. Nevertheless, GPS positioning works extremely poorly in most indoor scenarios, as the signals emitted by the GPS are too weak to penetrate most indoor building materials [3], and there are insurmountable obstacles in the line-of-sight transmission between satellites and mobile terminals; in addition, electromagnetic waves may be propagated in a non-line-of-sight manner, thus causing indoor positioning accuracy levels that cannot meet requirements or that are completely ineffective [7], [8]. Hence, an indoor positioning technique that can deal with the deficiencies of the GPS positioning system in a closed space has become an urgent demand. Although considerable indoor 
localization technologies have been developed to replace the application of GPS in indoor scenarios [4], [9], the availability of location information provided by these positioning techniques is still a vital element that limits the development of location-based services in indoor scenarios.

A wireless local area network (WLAN) has been considered to be one of the most popular approaches in indoor positioning systems [7], [9]-[11]. In addition, a received signal strength indicator (RSSI) has been regarded to be the signal source in most WLAN-based localization systems. This is because the 802.11 network of the WLAN can easily extract the RSSI of all wireless access points within the receiving range. Consequently, no additional software or hardware manipulation is required for WLAN infrastructures to implement positioning. Additionally, in the actual application process, RSSI-based WLAN localization technology also possesses the features of high-cost performance, strong expansibility and low energy consumption [4], [9], [12], which means that researchers have increasingly supported the use of indoor localization systems based on RSSI [13]-[15]. The literature has reported that the RSSI-based positioning accuracy under the current WLAN environment is maintained approximately within 2-5 m, [4] whereas in practice, most location-based services have higher requirements for accuracy and real-time performance [16], [17]. Therefore, it is urgent to further improve the real-time localization accuracy based on RSSI in WLAN environments, thus realizing its important engineering application value.

Due to an extremely complex indoor environment, the RSSI in WLAN is susceptible to the multipath effect, non-line-of-sight and shadow fading, which will generate low and unstable real-time localization accuracy [3], [16], [18]. Accordingly, many researchers have been devoted to the study of how to improve anti-jamming ability and real-time positioning accuracy for the localization algorithm. So far, RSSI-based indoor positioning techniques have been divided mainly into the range measurement and the location fingerprint [11], [19]. RSSI-based range measurements can estimate the distance between the emitter and the receiver through the path loss lognormal shadowing model of the wireless signal. However, the parameters of the path loss model are related to the characteristics of signal propagation in the environment, and the location environment presents a time-varying system [20].

Therefore, adopting a single environmental parameter will lose the self-adaptability of the path loss model. Some researchers have proposed a distributed real-time indoor localization framework based on RSSI and indicated that the anchor node can dynamically modify the internal parameters of the path loss model by periodically searching for nearby reference nodes and by improving the accuracy of distance estimation [16]. For a location fingerprint based on the RSSI, a fingerprint database can be established initially by acquiring the RSSI features of key nodes, and then the RSSI information obtained from the target location point can be matched by this fingerprinting database, with the location having the highest matching degree representing the position of the target location point. In addition, this localization technology of fingerprint can be considered to be a pattern recognition or machine learning problem [21], [22]. However, in fact, fingerprint localization technology also has some defects, such as the time-consuming data acquisition that is needed in the offline phase. Furthermore, due to signal propagation changes that are induced by environmental variation, the reliability of the collected data will be severely affected or will even no longer be valid. Thus, calibration processes in large amounts often have to be repeated to ensure location accuracy [9].

From the above, under an RSSI-based WLAN environment, both the range measurement and location fingerprint are usually vulnerable to the impact of complicated environmental conditions or environmental changes, thus resulting in imprecise positioning information. Herein, to elude these intractable issues, a novel real-time hybrid positioning algorithm was proposed based on the self-adaptive dynamic ranging model to address the problems of low and unstable indoor positioning accuracy. The rest of the paper is organized as follows. In Section 2, a self-adaptive dynamic ranging model is first proposed, which can 
update the environmental parameters and correct the ranging values of mobile terminals in real-time and can be combined with the maximum likelihood estimation to determine the prior probability of mobile terminal appearing at each key node. Then, a hybrid entirely weighted KNN algorithm (defined as H-EWKNN) is proposed based on the self-adaptive dynamic ranging model. Finally, another hybrid-weighted Bayesian algorithm (defined as H-WBayesian) is also presented by combining the self-adaptive dynamic ranging model with the Bayesian algorithm. In Section 3, first, the application framework and scenario of the positioning system are briefly introduced. Subsequently, the measurements of the path loss model and the location fingerprint data of key nodes were carried out in the real application scenario, and a total of 570 sets of real-time RSSI data from 19 test points were collected for algorithm verification. Second, the anti-jamming ability and ranging accuracy of the self-adaptive dynamic ranging model were further verified. Finally, both hybrid algorithms that are proposed in this paper were compared and verified for their anti-interference and positioning accuracies. The conclusions that were drawn from these results are explained in Section 4.

\section{Hybrid Algorithm for Real-Time Indoor Localization}

The method in this paper is to use a mobile terminal to measure the raw RSSI value of different access points for real-time positioning. First, the raw RSSI value is converted into distance information by the self-adaptive dynamic ranging model. Second, the residual function of each key node is obtained through the algorithm of the maximum likelihood estimate (MLE). Third, the distance value is used as the weight of each access point, and the residual function is used as the prior probability of each key node, which is further used for algorithm fusion with the k-nearest neighbor (KNN) algorithm and the Bayesian algorithm in fingerprint positioning technology, then in developing the H-EWKNN and H-WBayesian algorithms. Finally, both fusion algorithms are separately employed to locate the mobile terminal in real time

\subsection{Self-Adaptive Dynamic Ranging Model}

The propagation of the radio signal is often accompanied by a loss of energy intensity, and the loss of energy intensity and the propagation distance of the radio signal conform to the corresponding mathematical relationship. After the mobile terminal measures the strength of the radio signal emitted by the access point, the radio strength is further converted into communication distance by utilizing the path loss model [13], [15], [17], [23]. The strength of the radio signal declines as the propagated distance increases in the process of transmission, and the relationship between the communication distance and the radio signal strength can be expressed as:

$$
P L(d)=P L\left(d_{0}\right)-10 n \lg \frac{d}{d_{0}}+X_{\sigma}
$$

where $P L(d)$ and $P L\left(d_{0}\right)$ represent the power values of any position $\mathrm{d}$ and reference position $d_{0}$ respectively, and the unit of power value is decibel-milliwatts $(\mathrm{dBm})$ rather than milliwatts $(\mathrm{mW}) ; n$ represents the path loss factor, which is related to the specific propagation environment of the channel, as described in detail in the following section; and $X_{\delta}$ represents the Gaussian random variable with zero mean caused by shadow fading [24]. In the localization environment, the variance $\sigma$ of Gaussian random variables can be extensively tested to acquire, which is regarded as a constant [20].

The RSSI-based ranging method shows that the communication distance between the access points and the mobile terminals is calculated exactly by using Eq. (1). Then, the access point $i$ and the maximum likelihood estimate distance $\hat{d}_{i}$ of the mobile terminal can be defined as:

$$
\hat{d}_{i}=d_{0} 10^{\frac{R S I_{i}\left(d_{0}\right)-R S I_{i}(d)}{10 n}}
$$


where $\operatorname{RSSI}_{i}(d)$ and $\operatorname{RSSI}_{i}\left(d_{0}\right)$ are measured at any position $d$ and reference position $d_{0}$ respectively. The relationship between the distance $\hat{d}_{i}$ of the maximum likelihood estimation and the real distance $d_{i}$ is represented as:

$$
\hat{d}_{i}=d_{i} 10^{-\frac{X_{\sigma}}{10 n}}
$$

The expected value of the maximum likelihood estimation distance $\hat{d}_{i}$ can be expressed as [13], [15]:

$$
E\left(\hat{d}_{i}\right)=\frac{1}{\sqrt{2 \pi} \sigma} \int_{-\infty}^{\infty} d_{i} 10^{\frac{X_{\sigma}}{10 n}} e^{-\frac{X_{\sigma}}{2 \sigma^{2}}} d_{X_{\delta}}=d_{i} e^{\frac{\sigma^{2}}{\alpha^{2} n^{2}}}
$$

where $\alpha$ is equal to $10 / \ln (10)$. The maximum likelihood estimation distance $\hat{d}_{i}$ is a deviation estimate of real distance $d_{i}$. Thus, according to the Eq. (4), the distance $d_{i}$ of no deviation estimate can be denoted as:

$$
\tilde{d}_{i}=d_{0} 10 \frac{\operatorname{RSII}_{i}\left(d_{0}\right)-R S S I_{i}(d)}{10 n_{i}}-\frac{\sigma^{2}}{20 \alpha n_{i}{ }^{2}}
$$

where, the path loss factor $n_{i}$ represents the different propagation characteristics between the mobile terminal and the access point. Currently, a broad simplification is to assume that the path loss factor $n_{i}$ is the same constant [23], [25], [26]. However, in an indoor localization environment, this parameter possesses time-varying and unpredictable features, and the propagation characteristic of different access point is not completely the same.

The simplified way that a single path loss factor $n$ is used to represent a various path loss factor $n_{i}$ at different moments is an oversimplification [20]. Herein, this simplified approach is no longer adopted in this paper. Conversely, the path loss factor $n_{i}$ will be treated as a series of different variables, and it can be assumed that these variables are consistent over a very short period of time [20]. Then, two reference nodes defined as $C_{1}$ and $C_{2}$ are set in the positioning environment. The $R S S I_{i}$ value that is measured by the reference node in real time is substituted into Eq. (5). After that, the path loss factors $n_{i}$ of different access points can be dynamically updated at each moment, thus realizing the self-adaptive dynamic ranging process. When a distance $\tilde{d}_{i}$ exceeds the maximum radius $d_{\max }$ of the application scenario, indicating that the access point is severely disturbed by the environment, the self-adaptive dynamic range model will be the default distance $\tilde{d}_{i}$ to $d_{\max }$ to, further improve the anti-interference ability of the algorithm. The following text will verify that the self-adaptive dynamic ranging model proposed in this paper has higher ranging accuracy and positioning performance than does the simplified method of the single path loss model.

Location estimation is usually performed after obtaining the distance between the mobile terminal and the access point. As we know, the maximum likelihood estimation algorithm is a common statistical method based on the distance measuring principle, which is often used to solve indoor positioning problems [27], [28]. The assumption is that the number of access points is $N_{A P}$, the corresponding coordinate set is $A P_{i}=$ $\left(X_{i}, Y_{i}\right), i=1,2 \ldots N_{A P}$, and the real location for the mobile terminal is $(x, y)$. Then, the real distance $d_{i}$ between the mobile terminals and the access points can be denoted as:

$$
\left\{\begin{array}{c}
\left(X_{1}-x\right)^{2}+\left(Y_{1}-y\right)^{2}=d_{1}^{2} \\
\left(X_{2}-x\right)^{2}+\left(Y_{2}-y\right)^{2}=d_{2}^{2} \\
\vdots \\
\left(X_{N_{A P}}-x\right)^{2}+\left(Y_{n}-y\right)^{2}=d_{N_{A P}}^{2}
\end{array}\right.
$$

Then, the real distance and residual function of the estimated distance can be represented as:

$$
R_{i}(x, y)=\sqrt{\left(X_{i}-x\right)^{2}+\left(Y_{i}-y\right)^{2}}-\tilde{d}_{i}
$$


In the ideal case, when the sum of the squared residuals of the residual function is zero, the estimated position is the real position of the mobile terminal at the moment [20], [29]. The sum of the squared residuals of the residual function can be denoted as:

$$
S(x, y)=\sum_{i=1}^{N_{A P}} R_{i}(x, y)^{2}
$$

The maximum likelihood estimation algorithm is used to obtain the estimated position of the mobile terminal by minimizing the sum of squared residuals. Equation 7 is simplified into the form of $A X=B$, where $X=(x, y)^{T}$. Then,

$$
\begin{gathered}
A=2\left[\begin{array}{c}
X_{1}-X_{n} Y_{1}-Y_{n} \\
X_{2}-X_{n} Y_{2}-Y_{n} \\
\vdots \\
X_{n-1}-X_{n} Y_{n-1}-Y_{n}
\end{array}\right] \\
B=2\left[\begin{array}{c}
X_{1}^{2}-X_{n}^{2}+Y_{1}^{2}-Y_{n}^{2}+d_{n}^{2}-d_{1}^{2} \\
X_{2}^{2}-X_{n}^{2}+Y_{2}^{2}-Y_{n}^{2}+d_{n}^{2}-d_{2}^{2} \\
\vdots \\
X_{n-1}^{2}-X_{n}^{2}+Y_{n-1}^{2}-Y_{n}^{2}+d_{n}^{2}-d_{n-1}^{2}
\end{array}\right]
\end{gathered}
$$

By the means of the least residual sum of squares, the position of the mobile terminal can be expressed as [30]:

$$
X=\left(A^{T} A\right)^{-1} A^{T} B
$$

Although the maximum likelihood estimation algorithm presents a simple and feasible real-time localization algorithm, its accuracy cannot meet the high requirement of the current location-based service, and the accuracy of this method is described in detail below. Our idea is that the sum of squared residuals of the key nodes $R P_{j}=\left(x_{j}, y_{j}\right), j=1,2 \ldots N_{R P}$ is calculated by adopting the maximum likelihood estimation algorithm, which is treated as an evaluation index. The sum of squared residuals is smaller, which indicates that there is greater probability that this location is a real position.

\subsection{Hybrid KNN Algorithm Based on the RSSI}

Generally, the issue of indoor positioning can be considered to be a machine learning problem or pattern recognition problem [25]. With the rapid development of computers, computing resources no longer have been an obstacle to the development of machine learning. Thus, using machine learning algorithms to solve indoor positioning problem has become a research hotspot [24], [25], [31]. The KNN localization algorithm based on the RSSI has been divided mainly into two stages, including an offline and online stage [32]. The main task of the offline phase is to obtain the location information of each key node and the RSSI characteristics at the corresponding location to build a location fingerprint database. The corresponding equation is expressed as:

$$
D_{o f f, j}=\left(F_{o f f, j, 1}, \ldots, F_{o f f, j, N_{A P}}: R P_{j}=\left(x_{j}, y_{j}\right)\right), j=1,2 \ldots N_{R P}
$$

where $D_{o f f, j}$ represents all the data of key node $j$ in the offline database; $F_{o f f, j, N_{A P}}$ represents the RSSI value of access point $i$ measured by key node $j ; N_{A P}$ represents the numbers of the access point; $R P_{j}$ represents the location information of key node $j$; and $N_{R P}$ represents the amounts of key node. The completeness of this location fingerprint database is of great significance to the accuracy of indoor localization [33].

The primary mission of the online stage is to compare the obtained RSSI information $\left(F_{o n, 1}, \ldots, F_{o n, N_{A P}}\right)$ with the data in the location fingerprint database and to calculate the distance between them and each key 
node of the location fingerprint database [32], [33]. The corresponding equation is exhibited as follows:

$$
d\left(R P_{j}\right)=\left[\sum_{i=1}^{N_{A P}}\left(F_{o f f, j, i}-F_{o n, i}\right)^{q}\right]^{\frac{-1}{q}}, q>0
$$

where $q$ denotes the various types of distance. In the typical distance equation of KNN, the weights for each access point are assumed to be the same, thus assuming that the importance of each access point is the same. However, this fact was presented in Fig. 5, when the signal of an access point was seriously interfered, and the traditional calculation method directly ignores the sudden impact, which will lead to a large deviation in the positioning result. Consequently, to reduce the influence of such situations in positioning accuracy, improving the KNN algorithm becomes an effective method. At present, a common way to improve the KNN algorithm is to adopt the means of the weighted processing of $k$ nearest neighbors, which are abbreviated as a WKNN algorithm [34]. The weight is determined by the distance $d\left(R P_{j}\right)$ [33]. The related formula is represented as:

$$
\begin{gathered}
(\hat{x}, \hat{y})=\sum_{j=1}^{k} w_{j} R P_{j}, j=1, \ldots k \\
w_{j}=\frac{1 / d\left(R P_{j}\right)}{\sum_{n=1}^{k} d\left(R P_{j}\right)}
\end{gathered}
$$

However, due to the severely complicated indoor environment, wireless signals are vulnerable to sudden impact in the process of transmission. Especially with the increase of transmission distance, the possibility of multipath interference becomes greater [15]. In addition, the related paper indicated that in the RSSI-based positioning system, the RSSI values are reliable only when the mobile terminal is near the access point. Conversely, when the mobile terminal is far away from the access point, the obtained RSSI value is unreliable [30]. As a consequence, it was believed that the RSSI value that was measured by the mobile terminal contained important information on the access point. Here, a hybrid entirety-weighted KNN algorithm based on a self-adaptive dynamic ranging model was proposed, which was abbreviated as the H-EW KNN algorithm. Initially, the self-adaptive range model was used to change the real-time RSSI values that were measured by the mobile terminal into the distance $\tilde{d}_{i}$, and the method of distance weighting was then applied to calculate the distance $d\left(R P_{j}\right)$ of the key nodes. Subsequently, $d\left(R P_{j}\right)$ was sorted in ascending order to obtain the sequence $\omega_{k}$. Finally, the position estimation of the mobile terminal could be obtained by utilizing the mean square error $S(x, y)$, which is calculated by the maximum likelihood estimation as the weight of $k$ nearest neighbors. The equation of the H-EWKNN algorithm is denoted as:

$$
\begin{gathered}
d\left(R P_{j}\right)=\left[\sum_{i=1}^{N_{A P}} w_{i}\left(F_{\text {off }, j, i}-F_{\text {on }, i}\right)^{q}\right]^{\frac{-1}{q}} \\
w_{i}=\frac{1 / \tilde{d}_{i}}{\sum_{i=1}^{N} 1 / \tilde{d}_{i}} \\
\omega_{k}=\left\{R_{j}=\left(x_{j}, y_{j}\right): d\left(R P_{j}\right)\right\}, j=1, \ldots k \\
w_{j}=\frac{1 / S\left(R P_{j}\right)}{\sum_{i=1}^{k} 1 / S\left(R P_{j}\right)} \\
(\hat{x}, \hat{y})=\sum_{j=1}^{k} w_{j} R P_{j}, j=1, \ldots k
\end{gathered}
$$

where $k$ represents the number of the nearest neighbors. When $k$ is equal to 1 , it is denoted as the nearest neighbor algorithm (defined as $\mathrm{NN}$ ). When $k$ is greater than 1 , it is denoted as the nearest neighbor algorithm of $k$ (defined as KNN). Some researchers have indicated that the accuracy of indoor positioning cannot be improved with the increase in the $k$ value [32]. To determine the obtained value of this parameter, the positioning accuracy under different obtained values will be discussed later, and this 
conclusion will be compared and verified.

\subsection{Hybrid Bayesian Algorithm Based on the RSSI}

To verify whether this method of a fusion KNN algorithm has certain adaptability, this work also proposes a new hybrid weighted Bayesian algorithm (defined as H-WBayesian) based on the self-adaptive dynamic ranging model, maximum likelihood estimation algorithm and Bayesian algorithm. The traditional Bayesian algorithm is a typical probabilistic technique, which has been widely applied in the field of indoor positioning [24], [31], [35], [36]. The principle of the traditional Bayesian algorithm is to preset a certain number of position nodes and give the prior probability distribution of these position nodes and the conditional probability of the position feature. After obtaining the current features, mobile terminals can obtain the posterior probability of different position nodes by reverse-conversion of the Bayesian network. The position with the largest posterior probability is the result of position estimation. An RSSI-based Bayesian positioning algorithm has been divided into offline and online stages [24], [31]. The main task of the offline phase is to obtain the location information of each key node and the RSSI characteristics at the corresponding location to build a location fingerprint database. The corresponding equation is expressed as:

$$
D_{o f f, j}=\left\{O_{o f f, j, N_{A P}}, \cdots, O_{o f f, j, N_{A P}}\right\}: R P_{j}=\left(x_{j}, y_{j}\right),=1,2 \ldots N_{R P}
$$

where $\left\{O_{o f f, j, N_{A P}} \cdots, O_{o f f, j, N_{A P}}\right\}$ denotes the sequence of observed values corresponding to each key node. The RSSI information that is obtained during the online phase presents $\left(O_{o n, 1}, \ldots, O_{o n, N_{A P}}\right)$. The basic formula of the Bayesian algorithm is defined as [24], [31]:

$$
P\left(R P_{j} \mid O_{o n}\right)=\frac{P\left(R P_{j}\right) P\left(O_{o n} \mid R P_{j}\right)}{P\left(O_{o n}\right)}
$$

where $P\left(R P_{j} \mid O_{o n}\right)$ represents the probability of the mobile terminal appearing at the position $R P_{j}$ under the condition of obtaining the new observation value $O_{o n}$, which is called the posterior probability; $P\left(O_{o n}\right)$ represents the probability distribution of the observation value. Since this value is independent of the position $R P_{j}$, it can be regarded as a constant when only the relative probability of the posterior probability is required to be solved [24], [31]; $P\left(R P_{j}\right)$ denotes the probability of appearing at position $R P_{j}$; namely, the prior probability. $P\left(O_{o n} \mid R P_{j}\right)$ is called the conditional probability or the likelihood function, which represents the observation probability of different access points at the specified position. It is assumed that the measured values $O_{o n, N_{A P}}$ of each access point are independent, and the likelihood function in Eq. (22) can be expressed as [37]:

$$
P\left(O_{o n} \mid R P_{j}\right)=\prod_{i=1}^{N_{A P}} P\left(O_{o n, i} \mid R P_{j}\right)
$$

In the likelihood function of this traditional Bayesian algorithm, the weight of each access point is considered to be the same. However, the reliability of each access point, which was discussed in section 2.2, is different; that is, when the mobile terminal is far from the access point, the RSSI value that was obtained is very unreliable. Accordingly, a hybrid geometric-weighted Bayesian algorithm that is based on the self-adaptive dynamic ranging model is presented, which is called the H-WBayesian algorithm. This algorithm is described as the self-adaptive ranging model that is first used to change the RSSI values that were measured by the mobile terminal into distance $\tilde{d}_{i}$, and then the distance weighting method is used to calculate the likelihood function. Finally, the estimated position of the mobile terminal can be obtained by using the mean square error $S(x, y)$ of the maximum likelihood estimation method as the prior probability by which to estimate the position. The likelihood function of the H-WBayesian algorithm is expressed as: 


$$
\begin{aligned}
P\left(O_{o n} \mid R P_{j}\right) & =\prod_{i=1}^{N_{A P} P} P\left(O_{o n, i} \mid R P_{j}\right)^{w_{i}} \\
w_{i} & =\frac{1 / \tilde{d}_{i}}{\sum_{i=1}^{N_{A P}} 1 / \tilde{d}_{i}}
\end{aligned}
$$

where $P\left(O_{o n, i} \mid R P_{j}\right)$ denotes the probability of $O_{o n, i}$ obtained by measuring the observed value of the access point $A P_{i}$ at the location $R P_{j}$, and the Gaussian radial kernel function is adopted to describe the likelihood function of all observed values [24].

$$
P\left(O_{o n, i} \mid R P_{j}\right)=\frac{1}{\sqrt{2 \pi} \sigma_{j, i}} e^{-\frac{\left(o_{i}-\mu_{j, i}\right)^{2}}{2 \sigma_{j, i} i^{2}}}
$$

where $\mu_{j, i}$ and $\sigma_{j, i}$ respectively represent the mean and the variance of the sequence $\left\{O_{o f f, j, N_{A P}}\right\}$ of the observation values of access point $A P_{i}$, which was obtained at position $R P_{j}$ in the fingerprint database. As shown in Fig. 3, the nonlinear graph of the Gaussian radial kernel function is drawn by using the mean of the observed values. It is assumed that all observations conform to the Gaussian distribution, and Gaussian noise with the mean value of zero and a variance of 1 is added to all observations.

The prior probability $P\left(R P_{j}\right)$ is usually simplified as the same value [36]. This simplification will result in the likelihood function $P\left(O_{o n} \mid R P_{j}\right)$, which directly determines the distribution of the posterior probability $P\left(R P_{j} \mid O_{o n}\right)$, thus determining the estimated position of the mobile terminal. Even so, this simplification will still lose some important information. For this problem, the residual sum of squares of each anchor node are obtained by adopting the maximum likelihood estimation based on the self-adaptive ranging model, to determine the prior probability of each key node. The related equation is presented as:

$$
P\left(R P_{j}\right)=\frac{1 / S\left(R P_{j}\right)}{\sum_{j=1}^{N_{R} P} 1 / S\left(R P_{j}\right)}
$$

According to Eqs. (22) to (27), the RSSI-based H-WBayesian algorithm that was proposed in this study can be represented as:

$$
P\left(R P_{j} \mid O_{\text {on }}\right)=\frac{1 / S\left(R P_{j}\right)}{\sum_{j=1}^{N_{R} P} 1 / S\left(R P_{j}\right)} \prod_{i=1}^{N_{A P}} P\left(O_{o n, i} \mid R P_{j}\right)^{w_{i}}
$$

The $R P_{j}$ corresponding to the maximized posterior probability is the estimated location of the mobile terminal.

\section{Indoor Localization Results}

\subsection{The Framework and Scene of the Localization System}

To verify the real-time hybrid positioning algorithm based on the self-adaptive dynamic ranging model, a set of positioning systems based on the RSSI was established. Fig. 1 illustrated the application framework that could realize the hybrid algorithm for real-time positioning, which could be divided into the client and the server. The client first obtained the fingerprint database of key locations through an offline collection and uploaded the data to the server database. When the client sent a positioning request, it uploaded the collected RSSI data to the server. After the server obtained the data that was sent by the client, it fetched the data of the database through instructions and sent the positioning result to the client. Then, a positioning scenario related to the application framework was presented in Fig. 2. This scenario included a total of 6 access points, 2 calibration points and a mobile terminal, of which, the acquisition terminal of the DG201 group motion was used as an access point, which owned functions such as a $433 \mathrm{MHz}$ wireless two-way communication,WIFi@2.4G wireless communication, sound-light prompt and OLED screen displaying the 
terminal running state, etc. Moreover, the wireless communication range exceeded $50 \mathrm{~m}$. Then, the S3 bracelet was used as the calibration point and the mobile terminal, based on a 32-bit RISC system processor and worked at a $32 \mathrm{MHz}$ main frequency. The system was powered by a $3.7 \mathrm{v}$ polymer lithium battery, and the principle is that the lithium battery needs to go through a power management chip--a low-voltage differential linear voltage regulator (LDO)--to charge the whole system. In addition, the system was integrated with a $433 \mathrm{M}$ radio-frequency circuit, and the radio frequency hardware based on $433 \mathrm{M}$ was equipped with a self-defined wireless communication protocol. This protocol enabled the terminal to search all the signals of access points within $200 \mathrm{~ms}$, and the signal strength and time information of the searched access point were sent to the computer through the serial port for storage and analysis.

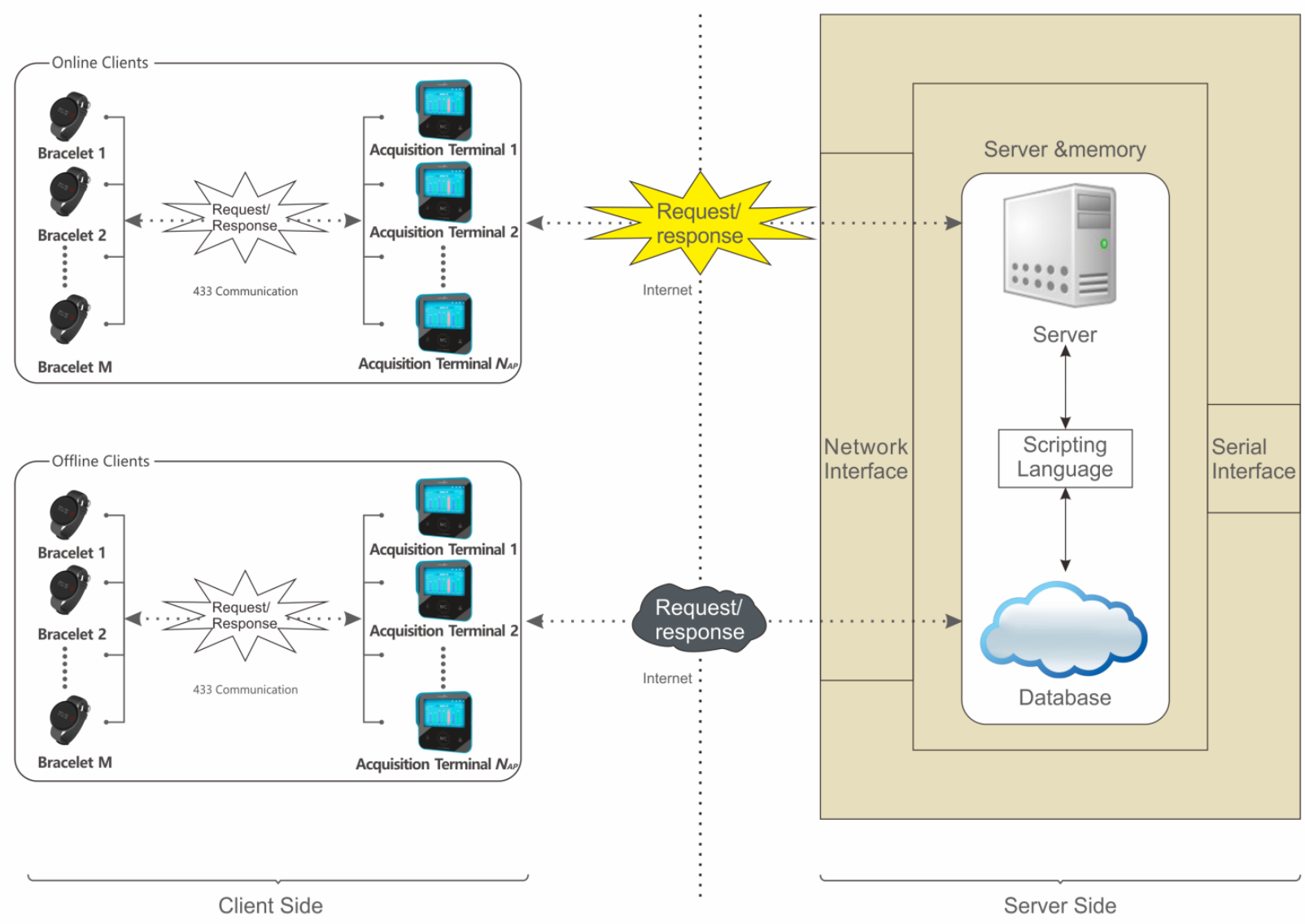

Fig. 1. The application framework of the hybrid algorithm for real-time positioning.

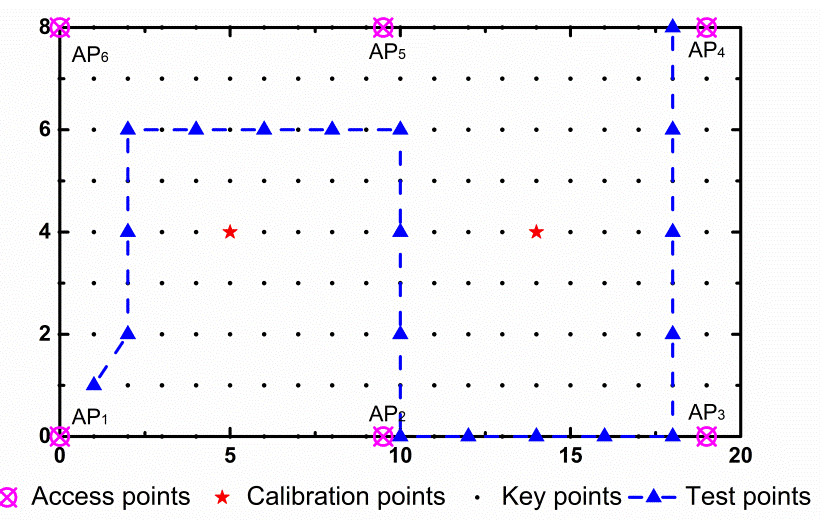

Fig. 2. The layout of the actual positioning scenario. 


\subsection{Data Acquisition for the Localization Algorithm}

Next, the offline data collection was first carried out in the real application scenario to build the location fingerprint database. 180 key nodes were selected in the positioning scenario, and 100 sets of RSSI data were then recorded at the locations of each key node (Fig. 2.). The data distribution of the different access points at various key node locations is shown in Fig. 3. It could be viewed that with the increase of distance, the RSSI value presented a trend of an overall decrease, and the fluctuation intensity of the RSSI value also gradually increased, which further confirmed the conclusion that was mentioned in section 2.2 that the RSSI value was more likely to be interfered with by the increase of the transmission distance. Subsequently, the online data collection included 19 test points that were selected on the planned path. Then, 30 sets of data were measured at each test point, which could be used to validate the self-adaptive dynamic ranging model and the two fusion algorithms. As shown in Fig. 4, the RSSI values of different access points were measured by the mobile terminal at the measured point $(1,1)$. It was found that in this test point, only the RSSI value of the AP4 manifested fluctuation due to an external interference, while the RSSI value of other access points without external interference remained relatively stable.

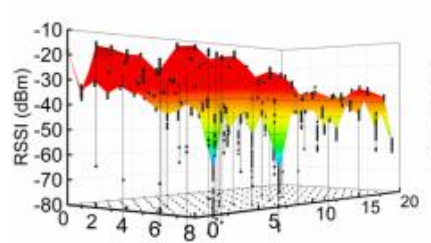

(a) $\mathrm{AP}_{1}$

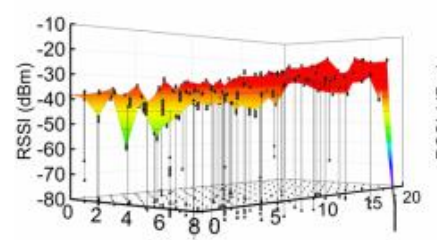

(d) $\mathrm{AP}_{4}$

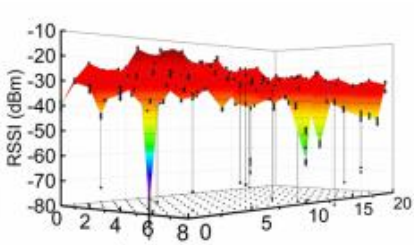

(b) $\mathrm{AP}_{2}$

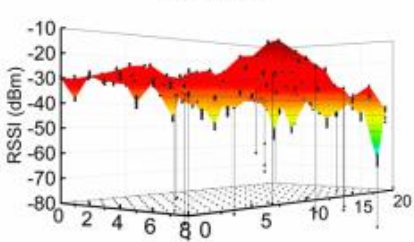

(e) AP5

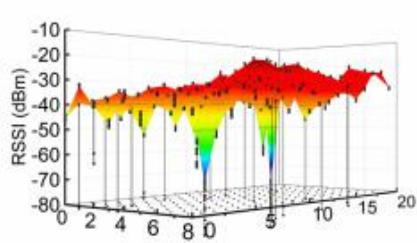

(c) $\mathrm{AP}_{3}$

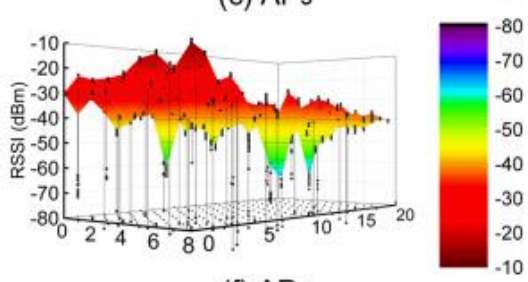

(f) AP6

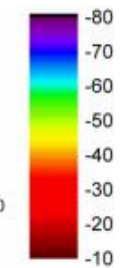

Fig. 3. The data distribution of different access points at various key node locations in the location fingerprint database.

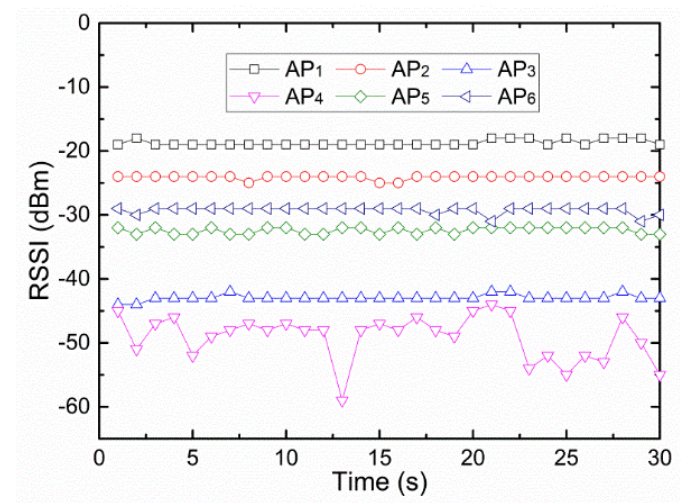

Fig. 4. The RSSI values of different access points at the measured point $(1,1)$.

\subsection{The Assessment of the Localization Performance}

To certify that whether the ranging accuracy and positioning performance in the self-adaptive dynamic ranging model presented higher than those in the single channel loss model [21]-[23], a single channel loss model was initially obtained by using the data in the location fingerprint database. Fig. 5 shows the 
distribution of the distance and the RSSI values in the location fingerprint database. A single channel loss model $\operatorname{RSSI}(d)=A-10 n \log _{10}(d)$ was developed by linear fitting, of which $A=-18.710$ and $n=$ 1.676. The R-square of the fitting result was 0.3361 , and the previous study has demonstrated that the closer the R-square is to 1 , the better the fitting result is [38]. These data indicated that the single channel loss model could not well represent the propagation characteristics of all access points. This observation was due to the limitation that, in addition to the different propagation characteristics of various access points mentioned in section 2.1, the influence of random variables is not considered in this model. Hence, in the self-adaptive dynamic loss model, the influence of random variables was considered and assessed. Furthermore, to obtain the distribution parameters of the random variables, the location fingerprint database and single channel loss model were used for calculation. Consequently, the distribution of the random variable $X_{\sigma}$ is shown in Fig. 6, where the random variables obeyed a Gaussian distribution of a zero mean and variance $\sigma=5.004$, which further validated the hypothesis proposed in section 2.3 that the observed values obeyed a Gaussian distribution with zero mean.

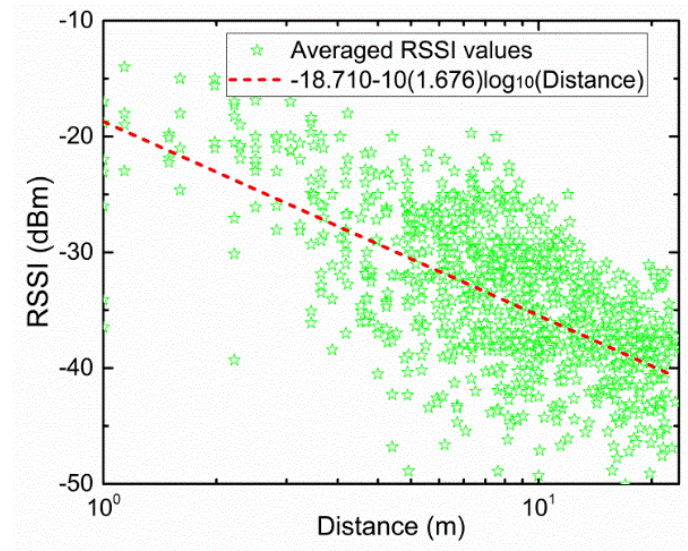

Fig. 5. The distribution between the distance and RSSI values in the location fingerprint database.

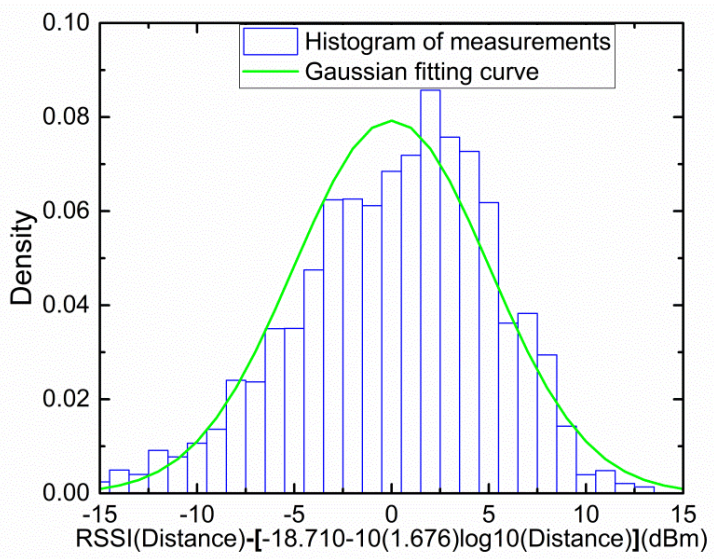

Fig. 6. The distribution of random variables with 108000 measurements in the positioning scenario.

Subsequently, the maximum likelihood estimation and the trilateration [3], [17], [27] based on both the single channel loss model and the self-adaptive dynamic ranging model were separately used to locate 19 test points in real time, which were further compared with the KNN and Bayesian algorithms. Furthermore, a more meaningful geometric position estimation error was adopted to assess the location estimation performance by calculating the location error $\varepsilon=\sqrt{(\hat{x}-x)^{2}+(\hat{y}-y)^{2}}$. As a result, Fig. 7 shows the comparison of the cumulative distribution function (CDF) of location error of 19 online measurement points 
calculated by different ranging methods. It could be clearly found that location estimation performance of the self-adaptive model was superior to that of the single loss model under the dual location algorithms. Meanwhile, the CDF calculated by different real-time positioning algorithms was also performed as shown in Fig. 8. Both the KNN and Bayesian algorithms based on fingerprint localization were better than the maximum likelihood estimation and the trilateration based on the self-adaptive dynamic ranging model. In summary, the above results showed that the self-adaptive dynamic ranging model could be conducive to improve location estimation performance, but the real-time location error still needed to be further reduced. By contrast, although the fingerprinting localization accuracy had certain enhancement, but it still stayed at the common error level of 2-5 $\mathrm{m}$. Hence, the combination of the self-adaptive dynamic ranging model and the fingerprinting method could be treated as one of the research directions to improve the real-time positioning estimation performance.

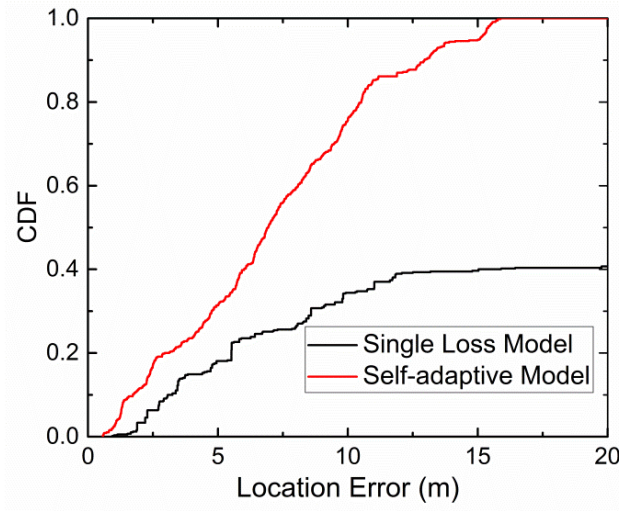

(a) Maximum likelihood estimation

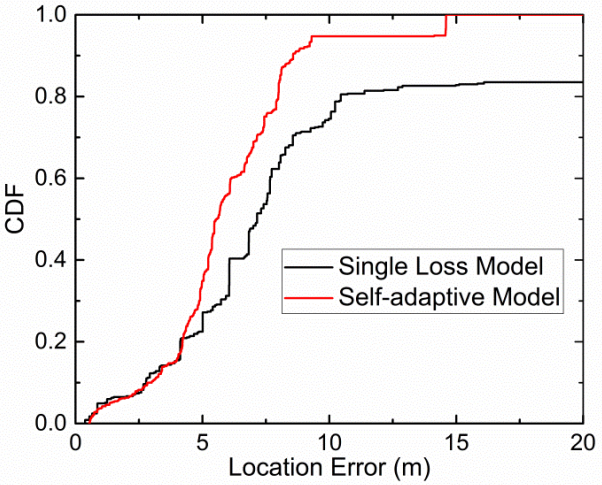

(b) Trilateration

Fig. 7. The distribution of random variables with 108000 measurements in the positioning scenario.

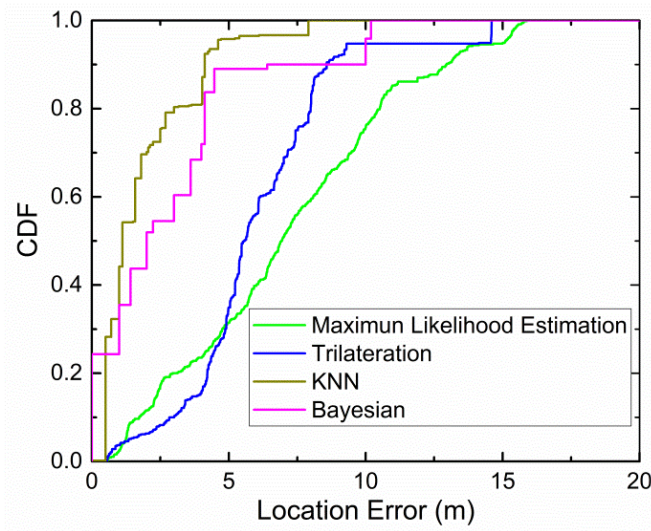

Fig. 8. The CDF of Curves of different real-time positioning algorithms.

\subsection{Real-Time Localization Results from the Hybrid Algorithm}

To verify whether the proposed hybrid algorithm based on a self-adaptive dynamic ranging model possesses certain high accuracy and anti-jamming ability, the H-EWKNN algorithm and the H-WBayesian algorithm presented in this study were applied for real-time positioning of the 19 online test points, and then compared with the KNN and Bayesian algorithms, respectively, For the verification of the high accuracy of hybrid algorithms, Fig. 9(a) shows the CDF of location error of different KNN algorithms, where $K=3$. It could be found that the location accuracy of H-EWKNN algorithm proposed was superior to that of 
the other KNN algorithms. In order to exclude the influence of different $K$ values on the above mentioned results, the overall average location error of the different KNN algorithms under the different $K$ values was investigated (Fig. 9(b)). Obviously, the positioning accuracy of H-EWKNN algorithm is the highest regardless of the K value, which reflected that the robustness of this hybrid algorithm under different values was also the best. Additionally, with the increase of the number of nearest neighbors, the overall average location error of the KNN and WKNN algorithms tended to first decrease and then increase. Conversely, the overall average location error of the H-WEKNN algorithm present gradual increase, which was consistent with the previous report [32]. Collectively, these outcomes suggested that the accuracy of indoor positioning could not be improved with the increase in the $\mathrm{K}$ value, so it was particularly important to select the appropriate number of nearest neighbors to improve the positioning accuracy. As a consequence, the number of nearest neighbors $K=2$ was determined by the H-EWKNN algorithm. In the meantime, the hybrid H-WBayesian algorithm was also compared with the results of the Bayesian algorithm. It can be found from Fig. 10 that H-WBayesian algorithm proposed was superior to the Bayesian algorithm. In general, the proposed hybrid algorithm can effectively improve the accuracy of indoor real-time positioning.

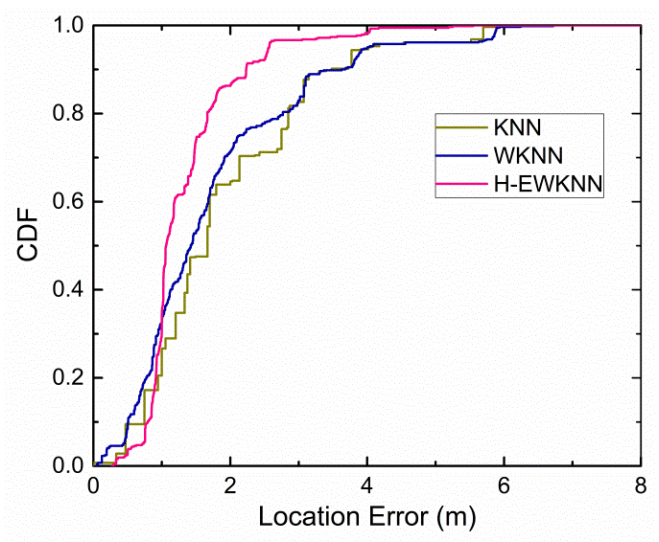

(a) The CDF

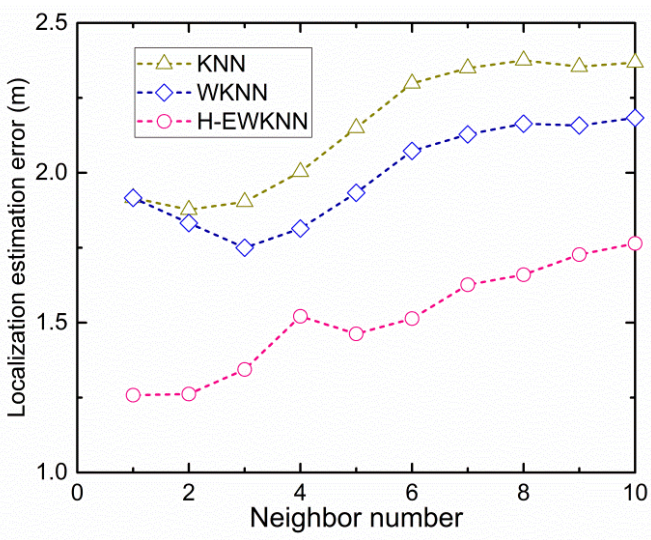

(b) The overall average location error

Fig. 9. The accuracy comparison of different KNN algorithms.

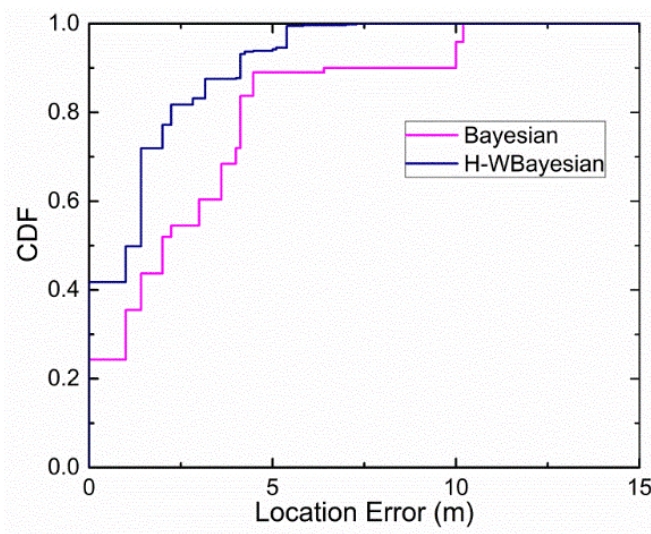

Fig. 10. The accuracy comparison of different Bayesian algorithms.

For the verification of the anti-jamming ability of hybrid algorithms, as shown in Fig. 11, the real-time positioning of 19 online test points is conducted by using different real-time positioning algorithms, and the standard deviation of location error of each test point was calculated. Transparently, the robustness of the two fusion algorithms exhibited significant improvement. 


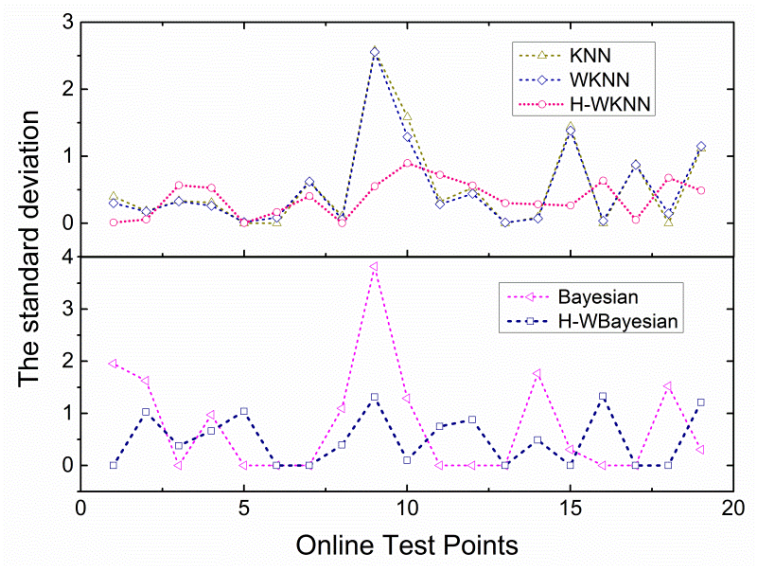

Fig. 11. Comparison of anti-interference capability of different real-time positioning algorithms.

Hybrid algorithm based on the self-adaptive dynamic ranging model was beneficial for improving the anti-jamming ability and positioning accuracy of the conventional real-time localization algorithm. In addition, the location error of the H-WBayesian algorithm was significantly lower than that of the H-EWKNN algorithm. Therefore, to further compare the positioning accuracy of both hybrid algorithms, two kinds of hybrid algorithms were utilized to locate the 19 test points in real time. As exhibited in Fig. 12, the average location error of dual hybrid algorithms for real-time location of different test points were assessed. The stability of the H-EWKNN algorithm was better than the H-WBayesian algorithm, in which the error rate of the H-EWKNN algorithm was lower than $3 \mathrm{~m}$ presented as $98.60 \%$, and the error rate of the $\mathrm{H}$-WBayesian algorithm was lower than $3 \mathrm{~m}$ remained at 83.16\%. In summary, these data demonstrated that the stability of the H-EWKNN algorithm was higher than that of H-WBayesian algorithm.

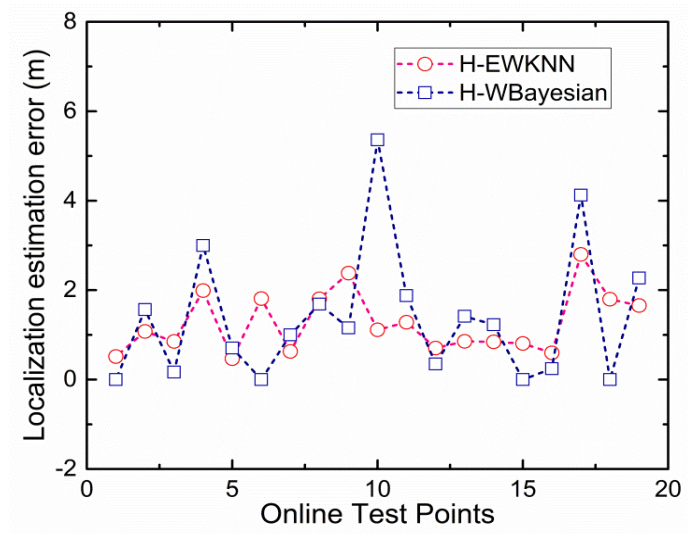

Fig. 12. The average location error of the dual hybrid algorithms for the real-time location of different test points.

Meanwhile, to confirm whether the proposed hybrid algorithm based on the self-adaptive dynamic ranging model could improve positioning accuracy, the maximum likelihood estimation, triangle centroid positioning, KNN, Bayesian, H-EWKNN and H-WBayesian algorithms were further used to locate 19 measured points in real time. Fig. 13 shows the comparison of the mean location error in 570 sets of data at 19 measured points under different real-time positioning algorithms. It was clearly observed that the average location error in the H-EWKNN algorithm was clearly reduced to $1.26 \mathrm{~m}$, which was $37.00 \%$ lower than that of KNN algorithm. The average location error of the H-WBayesian algorithm was reduced to 1.38 $\mathrm{m}$, which was 55.19\% lower than that of the Bayesian algorithm. From the above, these results suggested 
that the average location error in both the hybrid algorithms based on the self-adaptive dynamic ranging model remained in the range of 1.26-1.38 m, which showed significant improvement from the error level of 2-5 $\mathrm{m}$ in the current WLAN environment [4].

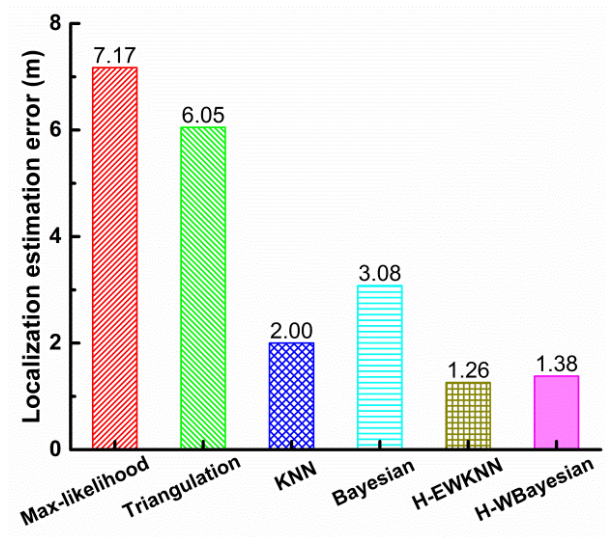

Fig. 13. The comparison of mean location error calculated by adopting different algorithms.

\section{Conclusion}

The new hybrid real-time localization algorithm based on a self-adaptive dynamic ranging model was developed in this paper. Through the self-adaptive dynamic ranging mode, the path loss factors of each access point were updated in real time, instead of the simplified way of using the same constant to represent the path loss factor, and, under the WLAN location environment, it was demonstrated that the observation values in this model obeyed the assumption of Gaussian distribution, and the accuracy and anti-interference ability of indoor localization were further strengthened. In addition, based on the above-mentioned model and the algorithms that were proposed by predecessors, a hybrid H-EWKNN algorithm and a hybrid H-WBayesian algorithm were proposed. Of these algorithms, both the stability and positioning accuracy in the H-EWKNN algorithm were better than those in the KNN and WKNN algorithms, respectively. At the same time, the indoor positioning accuracy could not be improved with the increase in the K value, and conversely, the location accuracy could be enhanced by selecting the appropriate number of nearest neighbors. Moreover, the stability and positioning accuracy of the H-WBayesian algorithm were also significantly improved compared with that of the conventional Bayesian algorithm. The range of the average location error of the above two hybrid algorithms maintained 1.26-1.38 m, which was significantly lower than the error level of 2-5 m under the current WLAN environment. Thus, these data indicated that this newly proposed hybrid algorithm based on the adaptive dynamic range model could effectively improve the indoor positioning stability and positioning accuracy with the real-time positioning algorithm, which provided a promising solution for the low accuracy and instability of indoor positioning caused by the RSSI susceptible to interference in WLAN.

\section{Conflict of Interest}

The authors declare that there are no conflicts of interest regarding the publication of this paper.

\section{Author Contributions}

Zhonghui Jang and Wu Huang conceived and designed this research work. Zhonghui Jang and Wei Xiao made substantial contribution in experimentation and data acquisition. Zhonghui Jang implemented the algorithm and analyzed the data. Dan Li and Defu Cheng provided professional assistance in 
experimentation. Xiao Wei and Zhonghui Jang wrote and revised this manuscript. All authors read and approved the final manuscript.

\section{Acknowledgment}

This work is supported by the 13th Five-Year Plan of the National Educational Science Key Issues of the Ministry of Education in 2017 under Grant No. DLA170428.

\section{References}

[1] Schiller, J., \& Voisard, A. (2004). Location-Based Services. San Francisco, CA, USA: Morgan Kaufmann.

[2] Flora, C., Ficco, M., Russo, S., et al. (2005). Indoor and outdoor location based services for portable wireless devices. Proceedings of the ICDCSW.

[3] Zhang, D., Xia, F., Yang, Z., et al. (2010). Localization Technologies for Indoor Human Tracking. Busan, South Korea: Futuretech.

[4] Yassin, A., Nasser, Y., Awad, M., et al. (2016). Recent advances in indoor localization: A survey on theoretical approaches and applications. IEEE Commun. Surv. Tut., 19(2), 1327-1346.

[5] Xu, G., \& Xu, Y. (2007). Applications of GPS theory and algorithms. Theory, Algorithms and Applications, 219-240.

[6] Fritsche, C., \& Klein, A. (2009). On the performance of hybrid GPS/GSM mobile terminal tracking. Proceedings of the ICCW.

[7] Gu, Y., Lo, A., \& Niemegeers. I. (2009). A survey of indoor positioning systems for wireless personal networks. IEEE Commun. Surveys Tuts., 11(1), 13-32.

[8] Seco, F., Jiménez, A. R., Prieto, C., et al. (2009). A survey of mathematical methods for indoor localization. Proceedings of the WISP.

[9] Farid, Z., Nordin, R., \& Ismail, M. (2013). Recent advances in wireless indoor localization techniques and system. J. Comput. Netw. Commun.

[10] Ismail, M. B., Fathi, A., Boud, A., et al. (2008). Implementation of location determination in a wireless local area network (WLAN) environment. Proceedings of the ICACT.

[11] Liu, H., Darabi, H., Banerjee, P., et al. (2007). Survey of wireless indoor positioning techniques and systems. IEEE Trans. Syst. Man Cybern. C Appl. Rev., 37(6), 1067-1080.

[12] Zàruba, G. V., Huber, M., Kamangar, F., et al. (2007). Indoor location tracking using RSSI readings from a single Wi-Fi access point. Wirel. Netw., 13(2), 221-235.

[13] Mao, G., Fidan, B., \& Anderson, B. D. (2007). Wireless sensor network localization techniques. Comput. Netw., 51(10), 2529-2553.

[14] Paul, A. S., \& Wan, E. A. (2009). RSSI-based indoor localization and tracking using sigma-point Kalman smoothers. IEEE J. Sel. Topics Signal Process., 3(5), 860-873.

[15] Mahapatra, R. K., \& Shet, N. (2018). Localization based on RSSI exploiting gaussian and averaging filter in wireless sensor network. Arabian J. Sci. Eng., 43(8), 4145-4159.

[16] Chen, W. C., Kao, K. F., Chang, Y. T., et al. (2018). An RSSI-based distributed real-time indoor positioning framework. Proceedings of the ICASI.

[17] Heinemann, A., Gavriilidis, A., Sablik, T., et al. (2014). RSSI-based real-time indoor positioning using ZigBee technology for security applications. Proceedings of the MCSS.

[18] Ren, Z., Huang, Y., Chen, Q., et al. (2009). Modeling and simulation of fading, pathloss, and shadowing in wireless networks. Proceedings of the ICCOMTA (pp. 335-343).

[19] Deak, G., Curran, K., \& Condell, J. (2012). A survey of active and passive indoor localisation systems. Comput. Commun., 35(16), 1939-1954. 
[20] Mazuelas, S., Bahillo, A., Lorenzo, R. M., et al. (2009). Robust indoor positioning provided by real-time RSSI values in unmodified WLAN networks. IEEE J. Sel. Topics Signal Process., 3(5), 821-831.

[21] Roos, T., Myllymäki, P., Tirri, H., et al. (2002). A probabilistic approach to WLAN user location estimation. Int. J. Wireless Inf. Netw., 9(3), 155-164.

[22] Tariq, O. B., Lazarescu, M. T., Iqbal, J., et al. (2017). Performance of machine learning classifiers for indoor person localization with capacitive sensors. IEEE Access, 5, 12913-12926.

[23] Bernhardt, R. (1987). Macroscopic diversity in frequency reuse radio systems. IEEE J. Sel. Area. Comm., 5(5), 862-870.

[24] Salo, J., Vuokko, L., El-Sallabi, H. M., et al. (2007). An additive model as a physical basis for shadow fading. IEEE Trans. Veh. Technol., 56(1), 13-26.

[25] Laitinen, H., Juurakko, S., Lahti, T., et al. (2007). Experimental evaluation of location methods based on signal-strength measurements. IEEE Trans. Veh. Technol., 56(1), 287-296.

[26] Li, X. (2006). RSS-based location estimation with unknown pathloss model. IEEE Trans. on Wirel. Commun., 5(12), 3626-3633.

[27] Goldoni, E., Savioli, A., Risi, M., et al. (2010). Experimental Analysis of RSSI-Based Indoor Localization with IEEE 802.15. 4. Lucca, Italy: EW.

[28] Sugano, M., Kawazoe, T., Ohta, Y., et al. (2006). Indoor localization system using RSSI measurement of wireless sensor network based on ZigBee standard. Proceedings of the 6th IASTED Int. Multi-Conf.Wireless Opt. Commun.

[29] Sotenga, P., Djouani, K., Kurien, A. M., et al. (2017). Indoor localisation of wireless sensor nodes towards internet of things. Procedia Computer Science, 109, 92-99.

[30] Vikas, C., Rajendran, S., Pattar, A., et al. (2016). WiFi RSSI and inertial sensor based indoor localisation system: A simplified hybrid approach. Proceedings of the ICONSIP (pp. 1-6).

[31] Carrasco, U., Coronado, P. D. U., Parto, M., et al. (2018). Indoor location service in support of a smart manufacturing facility. Comput. Ind., 103, 132-140.

[32] Xu, Y., Zhou, M., Meng, W., et al. (2010). Optimal KNN positioning algorithm via theoretical accuracy criterion in WLAN indoor environment. Proceedings of the GLOCOM (pp. 1-5).

[33] Shin, B., Lee, J. H., Lee, T., et al. (2016). Enhanced weighted K-nearest neighbor algorithm for indoor Wi-Fi positioning systems. Proceedings of the NCM and ICNIT (pp. 574-577).

[34] Zou, H., Huang, B., Lu, X., et al. (2016). Standardizing location fingerprints across heterogeneous mobile devices for indoor localization. Proceedings of the WCNC (pp. 1-6).

[35] Bekkali, A., \& Matsumoto, M. (2008). Bayesian sensor model for indoor localization in ubiquitous sensor network. Proceedings of the KINGN (pp. 285-292).

[36] Chen, L., Pei, L., Kuusniemi, H., et al. (2013). Bayesian fusion for indoor positioning using bluetooth fingerprints. Wireless pers. Commun., 70(4), 1735-1745.

[37] Gelman, A., Stern, H. S., Carlin, J. B., et al. (2013). Bayesian Data Analysis. New York, USA: Chapman and Hall.

[38] Gu, Y., \& Ren, F. (2015). Energy-efficient indoor localization of smart hand-held devices using bluetooth. IEEE Access, 3, 1450-1461.

Copyright (C) 2020 by the authors. This is an open access article distributed under the Creative Commons Attribution License which permits unrestricted use, distribution, and reproduction in any medium, provided the original work is properly cited (CC BY 4.0). 


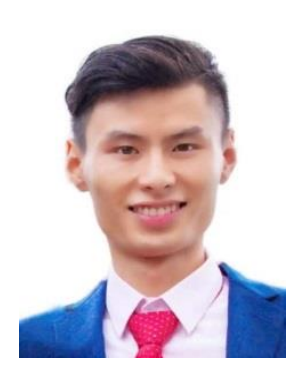

Zhonghui Jiang received a B.S. degree in mechanical engineering from Southwest Jiaotong University, China, in 2015, and M.S. degree in vehicle operation engineering from State Key Laboratory of Traction Power, Southwest Jiaotong University, China, in 2018. He currently works as an algorithm engineer in Chengdu Techman Software Co. LTD., China. His professional research interests are in data processing and analysis and research and application of machine learning algorithm.

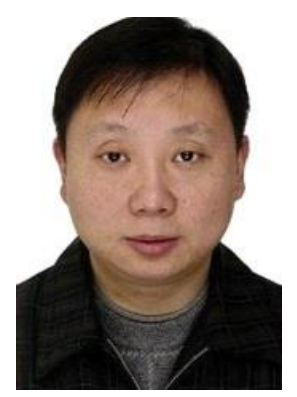

Wu Huang received a B.S. degree in computer science from Sichuan University, China, in 1993, and an M.S. degree in computer science from Sichuan University in 2000. He is currently pursuing a Ph.D. degree in the School of Computer Science at Sichuan University. He is a lecturer in the School of Computer Science at Sichuan University, China. His research interests include embedded real-time systems and intelligent medical instruments.

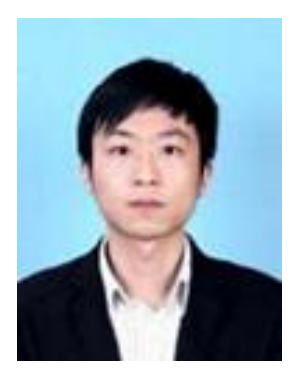

Xiao Wei received a B.S. degree in bioscience from Xichang College, China, in 2012. He received the Ph.D. degrees in materials science and engineering from Southwest Jiaotong University, China, in 2018. He is currently a senior research fellow in medicine and the manager of research department in Chengdu Techman Software Co. LTD., China. His current research interests mainly focus on the research and application of correlation algorithm for interior space location, and the study of the construction of multi-index system by using the mathematical statistics.

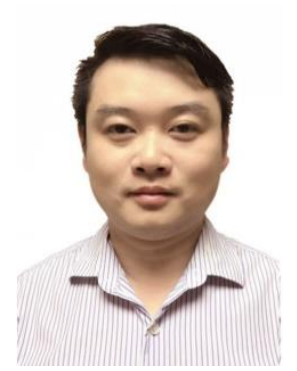

Defu Cheng received a B.S. degree in electronic and information engineering from Southwest University, China, in 2006. He received the M.S. degrees in electronic and information engineering from Southwest University, China, in 2009. From 2009 to 2019, $\mathrm{He}$ is currently a hardware engineer of research department in Chengdu Techman Software Co. LTD., China. His current research interests include smart wearable, signal acquisition system and medical devices.

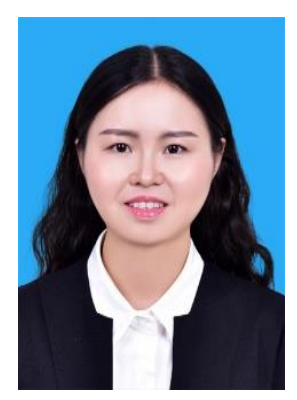

Dan Li received a B.S. degree in electronic information engineering from Xijing University, China, in 2014, and an M.S. degree in computer science from Guizhou Normal University in 2017. She currently works as an algorithm engineer in Chengdu Techman Software Co. LTD. Her current research interests include signal acquisition systems research and the application of machine learning algorithms. 